\title{
An interfacial Gerstner-type trapped wave
}

\author{
Jan Erik H. Weber \\ Department of Geosciences, University of Oslo, N-1315 Oslo, Norway
}

\begin{abstract}
Coastally trapped rotational interfacial waves are studied theoretically by using a Lagrangian formulation of fluid motion in a rotating ocean. The waves propagate along the interface between two immiscible inviscid incompressible fluid layers of finite depths and different densities, and are trapped at a straight wall due to the Coriolis force. For layers of finite depth, solutions are sought as series expansions after a small parameter. Comparison is made with the irrotational interfacial Kelvin wave. Both types of waves are identical to first order, having zero vorticity. The second order solution yields a relation between the vorticity and the velocity shear in the wave motion. Requiring that the mean motion in both layers is irrotational, then follows the wellknown Stokes drift for interfacial Kelvin waves. On the other hand, if the mean forward drift is identically zero, we obtain the second order vorticity in the Gerstner-type wave. The solutions in both layers for the Gerstner-type interfacial wave are given analytically to second order. It is shown that small density differences and thin upper layers both act to yield a shape of the material interfacial with broader crests and sharper troughs. These effects also tend to make the particle trajectories at the interface in both layers become distorted ellipses which are flatter on the upper side. It is concluded that the effect of air excludes the possibility of observing the exact Gerstner wave in deep water.

Keywords: Gerstner-type waves; Interfacial mode; Coastal trapping
\end{abstract}




\section{Introduction}

The Stokes edge wave [1] along a sloping beach can be described as a Gerstner wave [2]; see [3]. This is so even when the effect of the Earth's rotation is taken into account [4]. As shown in [4], in the limiting case when the sloping bottom becomes a vertical wall, the solution is a trapped barotropic Gerstner wave, which resembles the coastally trapped barotropic Kelvin wave. The trapped Gerstner solution, which is linear in wave amplitude, is an exact solution in Lagrangian coordinates for an infinitely deep ocean with a free material surface of constant pressure. This is not a common situation in geophysics, where gravity waves usually propagate along interfaces between immiscible fluids, like air and water for ocean waves. The presence of non-zero mass above the material oscillating boundary has the important consequence that the solutions in the air and in the water are restricted in amplitude. This is due to the fact that the material interface must be common for both layers, which requires that the wave steepness $\varepsilon$ must be small, and not just less than 1 as for the exact solution for one infinitely deep layer with a free surface (it should be noted that the Gerstner solution is three-dimensionally unstable [5] for $\varepsilon>1 / 3$ ). In addition, the exact Gerstner wave cannot exist in a fluid layer of finite depth [6]; see the discussion in [7]. A recent review of progressive irrotational surface waves in a layer of finite depth is given in [8]. It is pointed out that perturbation theory is not adequate for steep nonlinear waves. However, in the two-layer case with different densities and finite layer thicknesses, exact analytical solutions for Gerstner waves are not known. Accordingly, in this case approximate solutions may be attempted as series expansions after $\varepsilon$ as a small parameter.

Due to the Earth's rotation, a considerable amount of wave energy is trapped in coastal and equatorial regions within the Rossby radius of deformation. Recent papers on various aspects of equatorially trapped waves are numerous ([9]-[18]). Here we focus on coastal trapping. Since interfacial gravity waves play an important role in transferring energy in the ocean [19], it would 
be of interest to study Gerstner-type trapped coastal waves in a rotating fluid with a two-layer structure. In this connection the depth of both layers, as well as the density difference between the layers, will be of importance. This has motivated the present investigation.

The mathematical approach is based on the Lagrangian description of fluid motion. In this way we may compare our interfacial Gerstner-type trapped wave, which has zero mean particle drift, but possesses vorticity, to the more common irrotational interfacial Kelvin wave. The latter induces a mean forward Stokes drift [20]. We find that the solutions in Lagrangian coordinates are identical to first order, having zero vorticity; see also [7] for the non-rotating one-layer case. The second order solution yields a relation between the vorticity and the velocity shear in the interfacial wave motion. Requiring that the mean motion is irrotational, then follows the wellknown Stokes drift for interfacial Kelvin waves. On the other hand, if the mean forward drift is identically zero, we obtain the second order vorticity in the Gerstner-type wave. The solution for non-linear interfacial Gerstner-type trapped waves is given analytically, and the shape of the interface and the individual particle trajectories in each layer are discussed for various values of layer depths and densities.

The rest of this paper is organized as follows: In Section 2 we consider the mathematical formulation in Lagrangian coordinates, and in Section 3 we develop the solutions as series expansions after a small parameter. Section 4 contains analytical solutions of the trapped Gerstner-type interfacial wave, while in Section 5 we discuss the solutions for various values of layer depths and densities. Finally, Section 6 contains some concluding remarks.

\section{Mathematical formulation}

We study wave motion along the boundary between two horizontal inviscid, immiscible and incompressible fluid layers, denoting upper and lower layer variables by subscripts 1 and 2, 
respectively. When undisturbed, the layers have constant depths $H_{1}$ and $H_{2}$. The corresponding constant densities are $\rho_{1}$ and $\rho_{2}$, respectively, where $\rho_{2}>\rho_{1}$. The fluid layers are limited laterally by a straight vertical wall. We study the motion in a Cartesian coordinate system with the $x$-axis along the undisturbed interface and directed along the coastal wall, the $y$-axis points towards the sea, and the $z$-axis is vertically upwards. The system rotates with angular velocity $f / 2$ about the vertical axis, where $f$ is the constant Coriolis parameter. When disturbed, the material interface is given by $z=\xi(x, y, t)$.

The fluid motion is described by using a Lagrangian formulation. A fluid particle is identified by its Lagrangian coordinates $(a, b, c)$. The initial position of the particle is $\left(x_{0}, y_{0}, z_{0}\right)$, and the position at later times $t$ is $(x(a, b, c, t), y(a, b, c, t), z(a, b, c, t))$. The velocity components are $\left(x_{t}, y_{t}, z_{t}\right)$, where subscripts denote partial differentiation. The position of the material interface is given by $c=0$ for all times. Furthermore, we simplify, and assume that the upper and lower horizontal boundaries are impermeable, i.e. the vertical velocities vanish here.

Mass conservation (here volume conservation) requires that in each layer [21]

$$
D \equiv \frac{\partial(x, y, z)}{\partial(a, b, c)}=\frac{\partial\left(x_{0}, y_{0}, z_{0}\right)}{\partial(a, b, c)} .
$$

In the present analysis we consider trapped waves with particles moving in the $x, z$-plane, i.e. $y_{t}=0$. For such waves we can generally assume that $y=b$. The expression (1) then reduces to

$$
J\{x, z\}=J\left\{x_{0}, z_{0}\right\}
$$

where $J$ is the Jacobian operator defined by $J\{F, G\} \equiv F_{a} G_{c}-F_{c} G_{a}$.

In this case the equations of momentum in each layer can be written [21]: 


$$
\begin{aligned}
& x_{t t} x_{a}+z_{t t} z_{a}=-(p / \rho+g z)_{a}, \\
& x_{t t} x_{b}+z_{t t} z_{b}+f x_{t}=-(p / \rho+g z)_{b} \\
& x_{t t} x_{c}+z_{t t} z_{c}=-(p / \rho+g z)_{c},
\end{aligned}
$$

where $p$ is the pressure, $f$ the constant Coriolis parameter, and $g$ is the acceleration due to gravity.

\section{A series expansion solution}

Lagrangian perturbation solutions for surface waves with weak vorticity in a one-layer ocean of infinite depth have been discussed in [22] and [23]. For Gerstner type waves in a fluid layer of finite depth, an exact solution does not exist [6,7]. The same is of course true for the two-layer case. It should be noted that a two-layer non-rotating problem with a hydrostatic upper layer of constant speed, and an infinitely deep lower layer has been considered in [24]. But this problem differs very much from the case studied here. A standard procedure for the present problem is to consider the solutions in each layer as series expansions after a small parameter $\varepsilon$. For coastally trapped waves we can write

$$
\begin{aligned}
& x_{1,2}=a+\varepsilon x_{1,2}^{(1)}+\varepsilon^{2} x_{1,2}^{(2)}+. . \\
& y_{1,2}=b \\
& z_{1,2}=c+\varepsilon z_{1,2}^{(1)}+\varepsilon^{2} z_{1,2}^{(2)}+. . \\
& p_{1,2}=-\rho_{1,2} g c+\varepsilon p_{1,2}^{(1)}+\varepsilon^{2} p_{1,2}^{(2)}+. .
\end{aligned}
$$

see e.g. [25] for this approach in the Lagrangian formulation. Here we take that $\varepsilon=k A$ (the wave steepness), where $A$ is the interfacial wave amplitude and $k$ is the positive wave number.

For a one-layer model in a non-rotating fluid of finite depth such expansions for Gerstnertype waves have been carried out to third order in [26] for the restricted case that $D=1$ in (1), and generally to third order in [7]. For a rotating two-layer model, many of the basic features of the solution are obtained to second order (except the possible amplitude-dependence of the phase speed). Also, further analysis to higher orders involves a considerable amount of algebra. This 
labor could perhaps be better spent by applying numerical PDE solvers to this problem.

Therefore, in this analytical study, we truncate the series (4) after $O\left(\varepsilon^{2}\right)$.

For impermeable boundaries at $c=H_{1}$ and $c=-H_{2}$, trapped solutions to $O(\varepsilon)$ can be written

$$
\begin{aligned}
& x_{1,2}^{(1)}=\mu \frac{1}{k} \exp (-q b) \frac{\cosh k\left(c \mu H_{1,2}\right)}{\sinh k H_{1,2}} \cos \theta, \\
& z_{1,2}^{(1)}=\mu \frac{1}{k} \exp (-q b) \frac{\sinh k\left(c \mu H_{1,2}\right)}{\sinh k H_{1,2}} \sin \theta,
\end{aligned}
$$

where the minus sign in $\mu$ corresponds to the upper layer labelled 1 , where $c \in\left[0, H_{1}\right]$. In (5), $\theta=k a-\omega t$ is the phase function, where $\omega$ is the angular frequency. Furthermore, $q$ is the inverse Rossby radius of deformation. For details on water waves, the reader is referred to LeBlond and Mysak [27].

First we state some elementary facts for the linear problem. From (3) one finds that the continuity of pressure at the interface $c=0$ requires that

$$
\omega^{2}=\frac{g k\left(\rho_{2}-\rho_{1}\right)}{\rho_{1} \operatorname{coth} k H_{1}+\rho_{2} \operatorname{coth} k H_{2}} .
$$

From the linear vorticity equation in the seaward direction, it follows by inserting for $x_{1,2}^{(1)}, z_{1,2}^{(1)}$ that

$$
q=\frac{f k}{\omega}
$$

It is noted from (7) that to have coastally trapped solutions, i.e. $q>0$ when $b>0$, then $\omega$ in (6) must be positive, which means that the trapped interfacial wave must travel with the coast to the right in the northern Hemisphere.

From (3) we obtain for the momentum balance to second order: 


$$
\begin{aligned}
& x_{1,2 t t}^{(2)}+x_{1,2 t t}^{(1)} x_{1,2 a}^{(1)}+z_{1,2 t t}^{(1)} z_{1,2 a}^{(1)}=-\left(p_{1,2}^{(2)} / \rho_{1,2}+g z_{1,2}^{(2)}\right)_{a}, \\
& f x_{1,2 t}^{(2)}+x_{1,2 t t}^{(1)} x_{1,2 b}^{(1)}+z_{1,2 t t}^{(1)} z_{1,2 b}^{(1)}=-\left(p_{1,2}^{(2)} / \rho_{1,2}+g z_{1,2}^{(2)}\right)_{b}, \\
& z_{1,2 t t}^{(2)}+x_{1,2 t t}^{(1)} x_{1,2 c}^{(1)}+z_{1,2 t t}^{(1)} z_{1,2 c}^{(1)}=-\left(p_{1,2}^{(2)} / \rho_{1,2}+g z_{1,2}^{(2)}\right)_{c} .
\end{aligned}
$$

By differentiating with respect to time, (2) becomes

$$
x_{1,2 t a}^{(2)}+z_{1,2 t c}^{(2)}+\left(x_{1,2 a}^{(1)} z_{1,2 c}^{(1)}-x_{1,2 c}^{(1)} z_{1,2 a}^{(1)}\right)_{t}=0 .
$$

Inserting from (5), we obtain that

$$
\begin{aligned}
x_{1,2 t t}^{(2)} & +\frac{\omega^{2} \exp (-2 q b)}{2 k \sinh ^{2} k H_{1,2}} \sin 2 \theta=-\left(p_{1,2}^{(2)} / \rho_{1,2}+g z_{1,2}^{(2)}\right)_{a}, \\
f x_{1,2 t}^{(2)} & +\frac{q \omega^{2} \exp (-2 q b)}{k^{2} \sinh ^{2} k H_{1,2}}\left(\cosh ^{2} k\left(c \mu H_{1,2}\right) \cos ^{2} \theta+\sinh ^{2} k\left(c \mu H_{1,2}\right) \sin ^{2} \theta\right) \\
& =-\left(p_{1,2}^{(2)} / \rho_{1,2}+g z_{1,2}^{(2)}\right)_{b}, \\
z_{1,2 t t}^{(2)} & -\frac{\omega^{2}}{2 k \sinh ^{2} k H_{1,2}} \sinh 2 k\left(c \mu H_{1,2}\right)=-\left(p_{1,2}^{(2)} / \rho_{1,2}+g z_{1,2}^{(2)}\right)_{c} .
\end{aligned}
$$

Similarly, the volume conservation (9) reduces to:

$$
x_{1,2 t a}^{(2)}+z_{1,2 t c}^{(2)}+\frac{\omega \exp (-2 q b)}{\sinh ^{2} k H_{1,2}} \sin 2 \theta=0 .
$$

One interesting problem here is the fate of the vorticity, which is present in the exact Gerstner solution for infinite depth. In Eulerian notation the seaward vorticity $\zeta$ is defined as $\zeta=u_{z}-w_{x}$, where $u$ and $w$ are the horizontal and vertical velocity components. By applying the transformations

$$
R_{x}=J\{R, z\} / J\{x, z\}, \quad R_{z}=J\{x, R\} / J\{x, z\},
$$

we find that

$$
\zeta=\left(J\left\{x, x_{t}\right\}-J\left\{z_{t}, z\right\}\right) / J\{x, z\} .
$$

By inserting from (5), we have for the vorticity to $O(\varepsilon)$ :

$$
\zeta_{1,2}^{(1)}=x_{1,2 t c}^{(1)}-z_{1,2 t a}^{(1)}=0 .
$$


This means that the Gerstner-type wave to lowest order is identical to the irrotational interfacial Kelvin wave, as pointed out in [7] for the non-rotating one-layer case.

To second order (13) reduces to

$$
\begin{aligned}
\zeta_{1,2}^{(2)} & =x_{1,2 t c}^{(2)}-z_{1,2 t a}^{(2)}+x_{1,2 a}^{(1)} x_{1,2 t c}^{(1)}-x_{1,2 c}^{(1)} x_{1,2 t a}^{(1)}+z_{1,2 t c}^{(1)} z_{1,2 a}^{(1)}-z_{1,2 t a}^{(1)} z_{1,2 c}^{(1)} \\
= & x_{1,2 t c}^{(2)}-z_{1,2 t a}^{(2)}-\frac{\omega \exp (-2 q b)}{\sinh ^{2} k H_{1,2}} \sinh 2 k\left(c \mu H_{1,2}\right) .
\end{aligned}
$$

From the $x$ - and $z$-directions of (10) we obtain that

$$
x_{1,2 t c c}^{(2)}-z_{1,2 t t a}^{(2)}=0 .
$$

In Gerstner-type waves the motion is purely oscillatory (no mean drift), so for such waves it follows from (16) that $x_{1,2 t c}^{(2)}-z_{1,2 t a}^{(2)}=0$. From (15) we then obtain for the vorticity to $O\left(\varepsilon^{2}\right)$ for the Gerstner-type wave:

$$
\zeta_{1,2}^{(2)}=-\frac{\omega \exp (-2 q b)}{\sinh ^{2} k H_{1,2}} \sinh 2 k\left(c \mu H_{1,2}\right) .
$$

On the other hand, the interfacial Kelvin wave (the interfacial Stokes wave in a non-rotating ocean) is irrotational, and has a mean forward drift. By taking $\zeta_{1,2}^{(2)}=0$ in (15), averaging over the wave period, and assuming that the mean quantities does not vary in the horizontal direction, we find by integration in the vertical that

$$
\varepsilon^{2} \bar{x}_{1,2 t}^{(2)}=u_{1,2}^{S}=\frac{\varepsilon^{2} \omega \exp (-2 q b)}{2 k \sinh ^{2} k H_{1,2}} \cosh 2 k\left(c \mu H_{1,2}\right) .
$$

Here $u_{1,2}^{S}$ is the well-known Stokes drift in each layer for interfacial coastal Kelvin waves. We have put the integration constants equal to zero, since (18) also must be valid for infinitely deep upper and lower layers.

\section{Interfacial Gerstner-type waves to second order}


By definition the Gerstner-type wave has no forward mean drift, and is purely periodic in time. For such waves we may define a velocity potential $\varphi_{1,2}$ from $(16)$, where $x_{1,2}^{(2)}=\varphi_{1,2 a}$ and $z_{1,2}^{(2)}=\varphi_{1,2 c}$. The governing equation becomes from (11)

$$
\varphi_{1,2 a a}+\varphi_{1,2 c c}=-\frac{\exp (-2 q b)}{2 \sinh ^{2} k H_{1,2}} \cos 2 \theta
$$

Since we must have that $z_{1}^{(2)}=\varphi_{1 c}=0$ when $c=H_{1}$, and $z_{2}^{(2)}=\varphi_{2 c}=0$ when $c=-H_{2}$ we can write the solution

$$
\varphi_{1,2}=\exp (-2 q b)\left[\frac{1}{8 k^{2} \sinh ^{2} k H_{1,2}}+\frac{B_{1,2}}{k^{2}} \cosh 2 k\left(c \mu H_{1,2}\right)\right] \cos 2 \theta,
$$

where $B_{1,2}$ are dimensionless constants. Inserting for $x_{1,2}^{(2)}=\varphi_{1,2 a}$ and $z_{1,2}^{(2)}=\varphi_{1,2 c}$ from (19) into the $x$-component of (10), we now find for the $a$-dependent part of the pressure:

$$
\frac{p_{1,2}^{(2)}}{\rho_{1,2}}=\exp (-2 q b)\left(\frac{3 \omega^{2}}{4 k^{2} \sinh ^{2} k H_{1,2}}+\left[4 \omega^{2} \cosh 2 k\left(c \mu H_{1,2}\right)-2 g k \sinh 2 k\left(c \mu H_{1,2}\right)\right] \frac{B_{1,2}}{k^{2}}\right) \cos 2 \theta .
$$

We must require that $p_{1}^{(2)}=p_{2}^{(2)}$ along the material interface $c=0$. For this to be true, it is necessary that in (21)

$$
B_{1}=\frac{Q_{2}}{\kappa Q_{1}} B_{2}+\frac{3 \omega^{2}}{8 Q_{1}}\left(\frac{1}{\kappa \sinh ^{2} k H_{2}}-\frac{1}{\sinh ^{2} k H_{1}}\right)
$$

where

$$
\begin{aligned}
& \kappa=\rho_{1} / \rho_{2}<1, \\
& Q_{1}=2 \omega^{2} \cosh 2 k H_{1}+g k \sinh 2 k H_{1}, \\
& Q_{2}=2 \omega^{2} \cosh 2 k H_{2}-g k \sinh 2 k H_{2} .
\end{aligned}
$$


The final relation between $B_{1}$ and $B_{2}$ is obtained from the non-linear kinematic boundary condition at the material interface. From elementary Eulerian fluid mechanics we must have at the interface:

$$
w_{2}-w_{1}=\left(u_{2}-u_{1}\right) \frac{\partial \xi}{\partial x}, \quad z=\xi
$$

Linearly in Lagrangian coordinates, this means that $z_{1 t}^{(1)}=z_{2 t}^{(1)}$ at $c=0$. To $O\left(\varepsilon^{2}\right)$ we obtain

$$
z_{2 t}^{(2)}-z_{1 t}^{(2)}=\left(x_{2 t}^{(1)}-x_{1 t}^{(1)}\right) z_{1 a}^{(1)}, \quad c=0
$$

Inserting from (5), we find that

$$
z_{2 t}^{(2)}-z_{1 t}^{(2)}=\frac{\omega}{2 k}\left(\operatorname{coth} k H_{1}+\operatorname{coth} k H_{2}\right) \exp (-2 q b) \sin 2 \theta, \quad c=0
$$

Using (20) we then obtain

$$
B_{2}=-\frac{1}{8 \sinh 2 k H_{2}}\left(8 B_{1} \sinh 2 k H_{1}-\operatorname{coth} k H_{1}-\operatorname{coth} k H_{2}\right)
$$

Inserting from (27) into (22), $B_{1}$ becomes:

$$
\begin{aligned}
B_{1}= & \frac{3 \omega^{2} \sinh 2 k H_{2}}{8\left(\kappa Q_{1} \sinh 2 k H_{2}+Q_{2} \sinh 2 k H_{1}\right)}\left(\frac{1}{\sinh ^{2} k H_{2}}-\frac{\kappa}{\sinh ^{2} k H_{1}}\right) \\
& +\frac{Q_{2}\left(\operatorname{coth} k H_{1}+\operatorname{coth} k H_{2}\right)}{8\left(\kappa Q_{1} \sinh 2 k H_{2}+Q_{2} \sinh 2 k H_{1}\right)}
\end{aligned}
$$

where $\kappa, Q_{1}$ and $Q_{2}$ are given by (23). The explicit expression for $B_{2}$ follows readily from (27).

We can now write the particle displacements in each layer to $O\left(\varepsilon^{2}\right)$ for our interfacial Gerstner-type trapped wave from (5) and (20):

$$
\begin{aligned}
x_{1,2}= & a+\varepsilon x_{1,2}^{(1)}+\varepsilon^{2} x_{1,2}^{(2)}=a \mu \frac{\varepsilon}{k} \exp (-q b) \frac{\cosh k\left(c \mu H_{1,2}\right)}{\sinh k H_{1,2}} \cos \theta \\
& -2 \frac{\varepsilon^{2}}{k} \exp (-2 q b)\left[\frac{1}{8 \sinh ^{2} k H_{1,2}}+B_{1,2} \cosh 2 k\left(c \mu H_{1,2}\right)\right] \sin 2 \theta,
\end{aligned}
$$


and

$$
\begin{aligned}
z_{1,2}= & c+\varepsilon z_{1,2}^{(1)}+\varepsilon^{2} z_{1,2}^{(2)}=c \mu \frac{\varepsilon}{k} \exp (-q b) \frac{\sinh k\left(c \mu H_{1,2}\right)}{\sinh k H_{1,2}} \sin \theta \\
& +2 \frac{\varepsilon^{2}}{k} B_{1,2} \exp (-2 q b) \sinh 2 k\left(c \mu H_{1,2}\right) \cos 2 \theta
\end{aligned}
$$

As pointed out in [7] for the non-rotating one-layer case, Stokes-type expansions of the form (4) are not very efficient in shallow water $\left(k H_{1,2}<<1\right)$. For infinitely deep layers, i.e. $k H_{1,2} \rightarrow \infty$, we have $\omega=\{(1-\kappa) g k /(1+\kappa)\}^{1 / 2}$ from (6). In that case our solutions reduce to

$$
\begin{aligned}
& x_{1}=a-\frac{\varepsilon}{k} \exp (-q b-k c) \cos \theta-\frac{\varepsilon^{2}}{k} \frac{(1-3 \kappa)}{4\left(1-\kappa^{2}\right)} \exp (-2 q b-2 k c) \sin 2 \theta \\
& z_{1}=c+\frac{\varepsilon}{k} \exp (-q b-k c) \sin \theta-\frac{\varepsilon^{2}}{k} \frac{(1-3 \kappa)}{4\left(1-\kappa^{2}\right)} \exp (-2 q b-2 k c) \cos 2 \theta
\end{aligned}
$$

and

$$
\begin{aligned}
& x_{2}=a+\frac{\varepsilon}{k} \exp (-q b+k c) \cos \theta-\frac{\varepsilon^{2}}{k} \frac{\left(1+3 \kappa-2 \kappa^{2}\right)}{4\left(1-\kappa^{2}\right)} \exp (-2 q b+2 k c) \sin 2 \theta \\
& z_{2}=c+\frac{\varepsilon}{k} \exp (-q b+k c) \sin \theta+\frac{\varepsilon^{2}}{k} \frac{\left(1+3 \kappa-2 \kappa^{2}\right)}{4\left(1-\kappa^{2}\right)} \exp (-2 q b+2 k c) \cos 2 \theta
\end{aligned}
$$

When $\kappa=\rho_{1} / \rho_{2}<<1,(31)$ and (32) represent the solutions in air and water for trapped short waves. To have a common interface at $c=0$, we must require that $\varepsilon<<1$ in these solutions.

\section{Effect of layer depths and density differences}

To compare with earlier investigations of Gerstner-type waves in a single layer of finite depth with a free surface, we take in our calculations that $\kappa=\rho_{1} / \rho_{2}=0.001$ (air above water), $H_{1}=700 \mathrm{~m}, H_{2}=10 \mathrm{~m}, \lambda=100 \mathrm{~m}$, and $\varepsilon=k A=0.06$. With these parameters the upper air layer is in practice infinitely thick. The result for the interface $c=0$ is displayed in Fig. 1.

Fig. 1 
We note from Fig. 1 that nonlinearity strengthens the tendency of the linear solution towards more peaked interfacial crests and flatter troughs. This is qualitatively the same shape as for the exact Gerstner solution for a single infinitely deep layer with a free surface.

In Fig. 2 we have displayed the trajectories for particles at the interface for this case. The displacements are scaled by the wave amplitude.

Fig. 2

We note from Fig. 2 that the linear trajectory in the upper, "infinitely" thick layer is a circle, and in the lower finite depth layer it is an ellipsis, as expected. The nonlinearity yields that the particle trajectory in the lower layer (blue solid curve) is a deformed ellipsis which is more flat near the bottom. This is similar to what one finds for Gerstner-type waves in a one-layer model with finite depth; see [7, Fig. 6].

Another immiscible two-layer set-up that is relevant in nature, is oil above water. In this case the upper layer is usually much thinner than the lower layer. Typically, for oil above water we have $\kappa=\rho_{1} / \rho_{2}=0.9$. For a laboratory-scale experiment, we now choose $H_{1}=0.2 \mathrm{~m}, H_{2}=1 \mathrm{~m}$ and $\lambda=1 \mathrm{~m}$. In Fig. 3 we have depicted the shape of the interface for this case when $\varepsilon=0.03$. Fig. 3

We note from Fig. 3 that the interface is broader and somewhat depressed at the crests, and sharper and deeper at the troughs, which is contrary to what we observe in Fig. 1. Obviously, flat crests and sharp troughs are due to the presence of the thinner upper layer. This also affects the particle trajectories at the interface in the upper and lower layer; see Fig. 4 below. Again, the displacements are scaled by the wave amplitude.

Fig. 4

We note from Fig. 4 that the trajectories in each layer from linear theory are ellipses with 
different major axes (dotted curves). The nonlinear effect is to distort the ellipses by displacing them downwards and flatten them at the top. This is picture is consistent with the surface shape displayed in Fig. 3.

However, it is an interesting fact that not only the thickness, but also the density of the upper fluid also contributes to the shape of the interface. This is evident from Fig. 5, where we have plotted the situation in Fig. 1 with an infinitely thick upper layer, but now with a much denser fluid than air on top. This setting could model the effect of water above a thin mud layer at the bottom.

Fig. 5

We note from Fig. 5 the resemblance with the surface shape in Fig. 3, and we realize that increasing upper layer density contributes to the flattening of the crests and the sharpening of the troughs.

\section{Concluding remarks}

The boundary conditions for inviscid rotational gravity waves along a free surface, and for waves along an interface between immiscible fluids of different densities are quite different. In the first case the only requirement is that the pressure is constant along the surface, which yields the exact Gerstner solution in Lagrangian coordinates (linear in wave amplitude, phase speed independent of amplitude) in an infinitely deep layer. In this connection it should be mentioned that the Gerstner solution in an infinitely deep layer is also valid when the Coriolis force is included; see e.g. [28, 29]. If we add an infinitely deep upper layer of smaller density, the situation changes drastically, as seen from the approximate solutions (31) and (32). Now it must be required that the pressure at the interface as well as the velocities normal to the interface must be the same in both fluids. To satisfy both these conditions, restrictions must be put on the wave 
steepness $\varepsilon$, which must be small.

The effect of finite depth on rotational waves along a free surface yields similar problems. In particular, it is impossible to obtain a constant pressure along the free surface for all permissible $\varepsilon$ when the depth is finite [6]. Hence, solutions must be sought as series expansions; see e.g. [7,

26]. The addition of an upper layer of finite depth and different density complicates this problem even further, as shown in this paper.

In practice, even if the density of air is about $1 / 1000$ of that of water, the effect of air above water cannot be neglected. Hence, it will not be possible to generate the exact Gerstner wave solution in the laboratory with air on top. Accordingly, small-amplitude rotational Gerstner-type waves and irrotational Stokes waves will be difficult to distinguish in laboratory experiments. One way to separate them is to measure the mean drift in the Stokes wave. However, this may not be an easy task, as shown by Monismith et al. [30]; see also the discussion in [31] which includes the effect of viscosity.

\section{Acknowledgements}

Financial support from the Research Council of Norway through the Grant 233901 (Experiments on waves in oil and ice) is gratefully acknowledged.

\section{References}

[1] G.G. Stokes, Report on recent researches in hydrodynamics, in Mathematical and Physical Papers, vol. 1, (1880) pp. 157-187, Cambridge Univ. Press, Cambridge, U. K.

[2] F. Gerstner, Theorie der Wellen samt einer daraus abgeleiteten Theorie der Deichprofile, Ann. Phys. 2 (1809) 412-455.

[3] A. Constantin, Edge waves along a sloping beach, J. Phys. A Math. Gen. 34 (2001) 9723- 
9731.

[4] J.E.H. Weber, A note on trapped Gerstner waves, J. Geophys. Res. 117 (2012) C03048, doi: 10.1029/2011JC007776.

[5] S. Leblanc, Local stability of Gerstner's waves, J. Fluid Mech. 506 (2004) 245-254.

[6] M.-L. Dubreil-Jacotin, Sur les ondes de type permanent dans le liquides hétérogènes, Accad. Naz. Lincei 15 (1932) 814-819.

[7] D. Clamond, On the Lagrangian description of steady surface gravity waves, J. Fluid Mech. 589 (2007) 433-454.

[8] A. Constantin, The flow beneath a travelling surface wave, J. Phys. A: Math. Theor. 48 (2015) doi:10.1088/1751-8113/48/14/143001.

[9] A. Constantin, An exact solution for equatorially trapped waves, J. Geophys. Res. Oceans 117 (2012) C05029, doi:10.1029/2012JC007879.

[10] A.-V. Matioc, B.-V. Matioc, On periodic water waves with Coriolis effects and isobaric streamlines, J. Nonlinear Math. Phys. 19 (2012) 89-103.

[11] D. Henry, An exact solution for equatorial geophysical water waves with an underlying current, Eur. J. Mech. B Fluids 38 (2013) 18-21.

[12] A. Constantin, Some nonlinear, equatorial trapped, nonhydrostatic internal geophysical waves, J. Phys. Oceanogr. 44 (2014) 781-789.

[13] J.E.H. Weber, K.H. Christensen, G. Broström, Stokes drift in internal equatorial Kelvin waves; continuous stratification versus two-layer models, J. Phys. Oceanogr. 44 (2014) 591-599. [14] D. Ionescu-Kruse, An exact solution for geophysical edge waves in the $\beta$-plane approximation, J. Math. Fluid Mech. 17 (2015) 699-706. 
[15] J.E.H. Weber, Eulerian volume transport induced by spatially-damped internal equatorial Kelvin waves, J. Phys. Oceanogr. 45 (2015) 1794-1803.

[16] D. Henry, Equatorially trapped nonlinear water waves in a $\beta$-plane approximation with centripetal forces, J. Fluid Mech. 804 (2016) R1, 11 pp.

[17] D. Henry, S. Sastre-Gomez, Mean flow velocities and mass transport for equatoriallytrapped water waves with an underlying current, J. Math. Fluid Mech. 18 (2016) 795-804.

[18] J.E.H. Weber, Equatorial Stokes drift and Rossby rip currents, J. Geophys. Res. Oceans, 122 (2017) 4819-4828.

[19] V. Vlasenko, N. Stashchuk, K. Hutter, Baroclinic Tides: Theoretical Modeling and Observational Evidence, Cambridge University Press, 2005.

[20] G.G. Stokes, On the theory of oscillatory waves, Trans. Cam. Phil. Soc. 8 (1847) 441-455.

[21] H. Lamb, Hydrodynamics, $6^{\text {th }}$ ed. Cambridge University Press, 1932.

[22] A.A. Abrashkin, Three-dimensional Gouyon waves, Fluid Dyn. 31 (1996) 583-587.

[23] A.A. Abrashkin, E. Pelinovsky, Lagrange form of the nonlinear Schrödinger equation for low-vorticity waves in deep water, Nonlinear Processes in Geophysics 24 (2017) 255-264.

[24] R. Stuhlmeier, Internal Gerstner waves: application to dead water, Applicable Analysis 93 (2014) 1451-1457.

[25] W.J. Pierson, Perturbation analysis of the Navier-Stokes equations in Lagrangian form with selected solutions, J. Geophys. Res. 67 (1962) 3151-3160.

[26] J. Kravtchenko, A. Daubert, Closed trajectory waves in a finite depth, La Houille Blanche 3 (1957) 408-429.

[27] P.H. LeBlond, L.A. Mysak, Waves in the Ocean, Elsevier, 1978.

[28] R.T. Pollard, Surface waves with rotation: an exact solution, J. Geophys. Res. 75 (1970) 
$5895-5898$.

[29] A. Constantin, S.G. Monismith, Gerstner waves in the presence of mean currents and rotation, J. Fluid Mech. 820 (2017) 511-528.

[30] S.G. Monismith, E.A. Cowen, H.M. Nepf, J. Magnaudet, L. Thais, Laboratory observations of mean flow under surface gravity waves, J. Fluid Mech. 573 (2007) 131-147.

[31] J.E.H. Weber, Do we observe Gerstner waves in wave tank experiments? Wave Motion 48 (2011) 301-309.

\section{Figures}

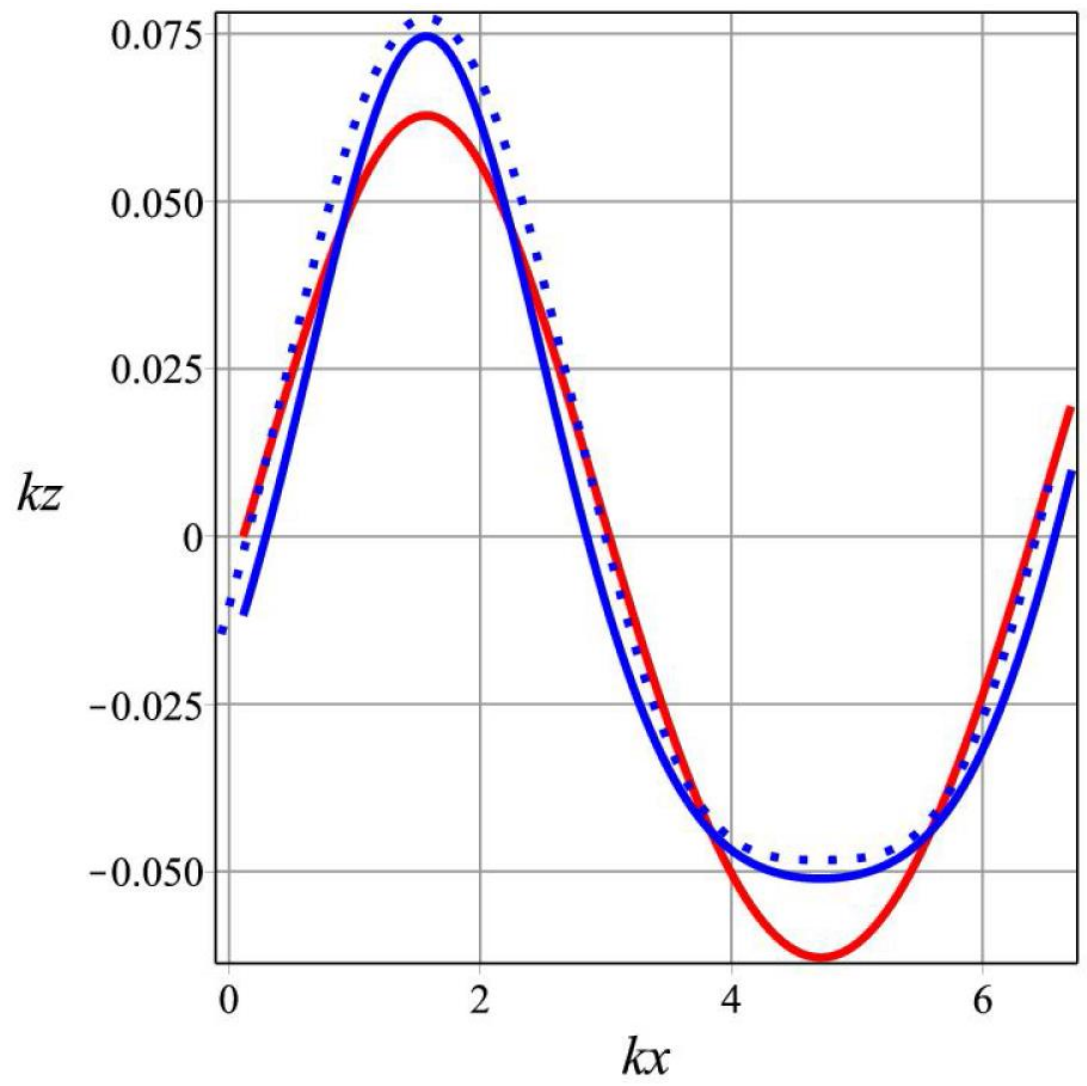

Fig. 1 . The interface at the vertical wall $(b=0)$ when $\rho_{1} / \rho_{2}=0.001, k H_{1}=44, k H_{2}=0.63$, and $\varepsilon=0.06$. Red curve: linear theory. Blue curve: nonlinear theory from lower layer solution. Blue dots: nonlinear theory from upper layer solution. 


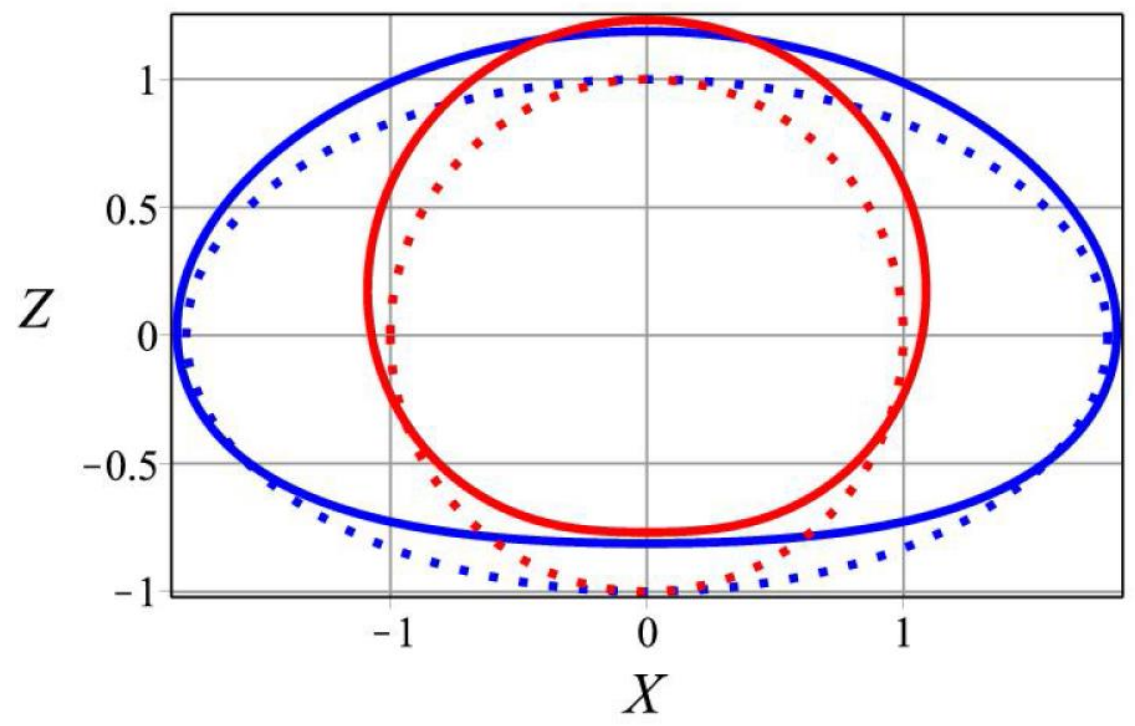

Fig. 2. Particle trajectories at the interface for the same parameters as in Fig. 1. Red solid curve: upper layer. Blue solid curve: lower layer. The dotted red and blue curves correspond to the linear solution in each layer. 


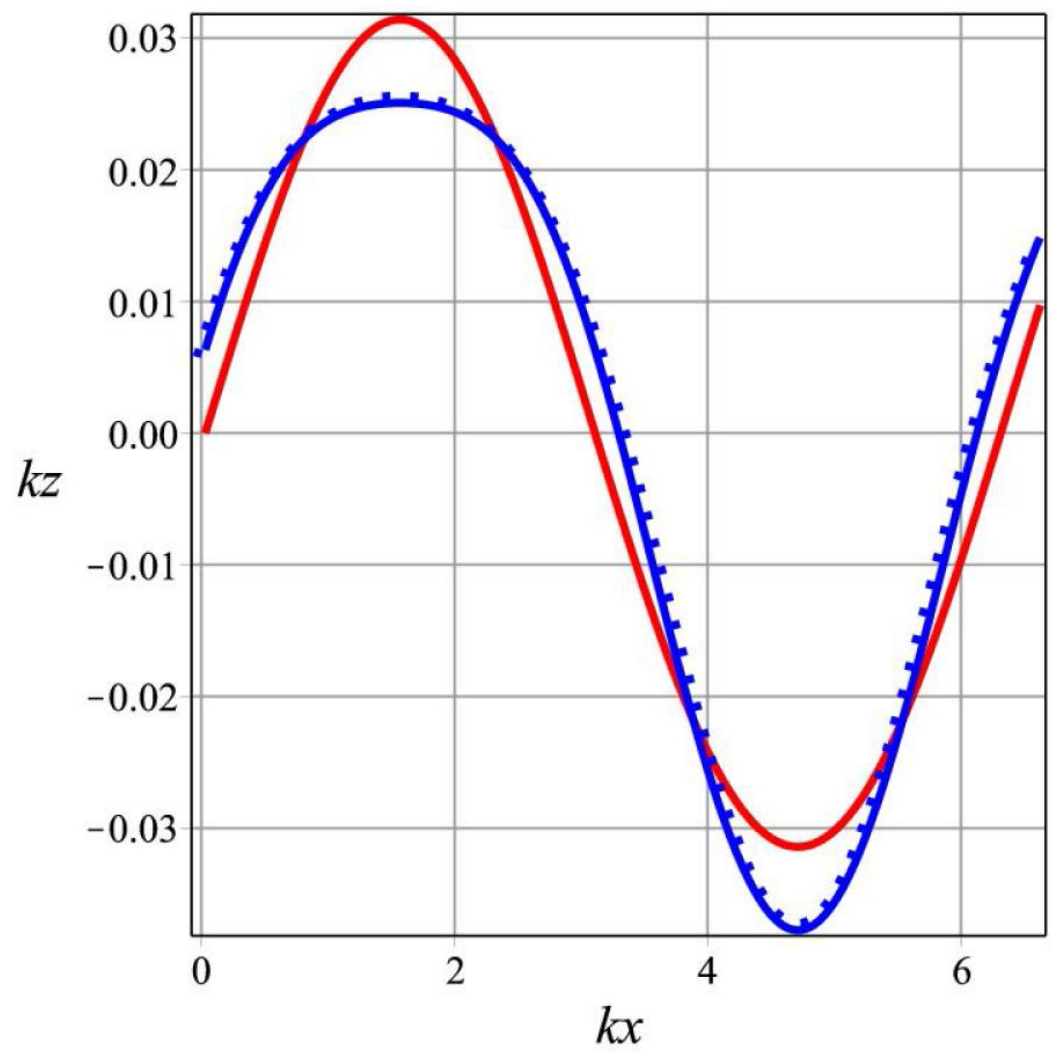

Fig. 3. The interface at the vertical wall $(b=0)$ when $\rho_{1} / \rho_{2}=0.9, k H_{1}=1.26, k H_{2}=6.28$, and $\varepsilon=0.03$. Red curve: linear theory. Blue curve: nonlinear theory from lower layer solution. Blue dots: nonlinear theory from upper layer solution. 


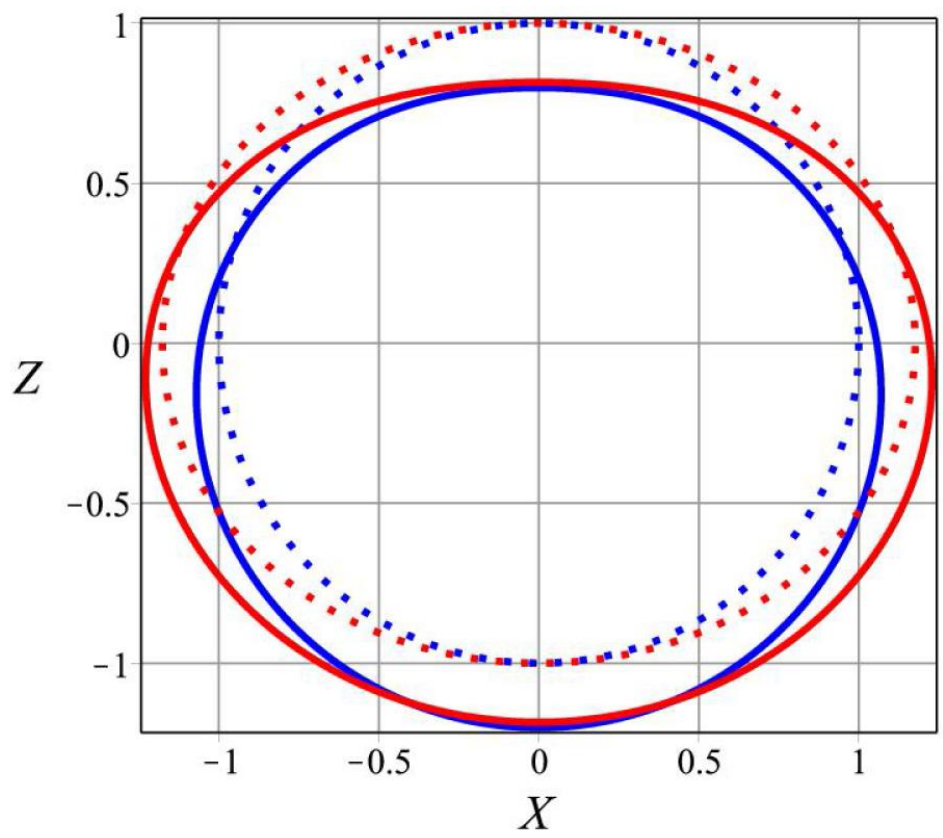

Fig. 4. Particle trajectories at the interface for the same parameters as in Fig. 3. Red solid curve: upper layer. Blue solid curve: lower layer. The dotted red and blue curves correspond to the linear solution in each layer. 


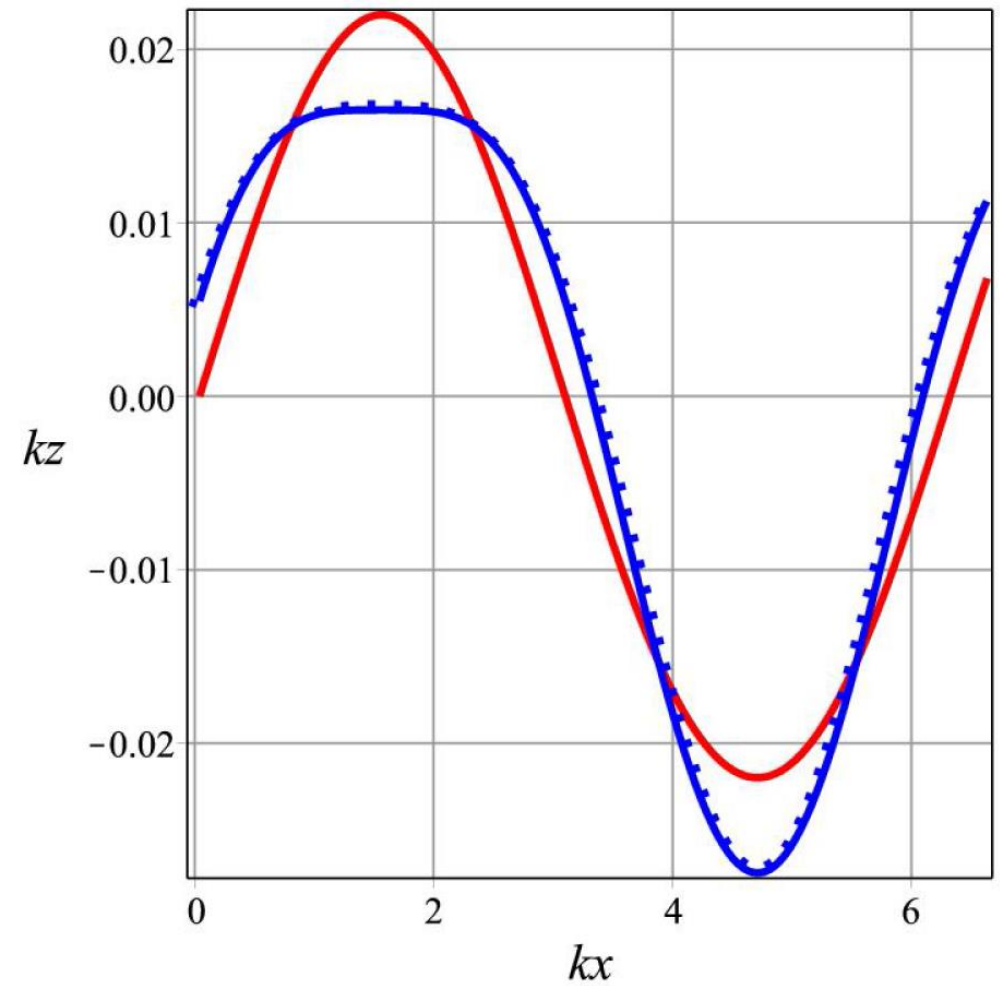

Fig. 5. The interface at the vertical wall $(b=0)$ when $\rho_{1} / \rho_{2}=0.9, k H_{1}=44, k H_{2}=0.63$, and $\varepsilon=0.022$. Red curve: linear theory. Blue curve: nonlinear theory from lower layer solution. Blue dots: nonlinear theory from upper layer solution. 


\section{Figure legends}

Fig. 1. The interface at the vertical wall $(b=0)$ when $\rho_{1} / \rho_{2}=0.001, k H_{1}=44, k H_{2}=0.63$, and $\varepsilon=0.06$. Red curve: linear theory. Blue curve: nonlinear theory from lower layer solution. Blue dots: nonlinear theory from upper layer solution.

Fig. 2. Particle trajectories at the interface for the same parameters as in Fig. 1. Red solid curve: upper layer. Blue solid curve: lower layer. The dotted red and blue curves correspond to the linear solution in each layer.

Fig. 3. The interface at the vertical wall $(b=0)$ when $\rho_{1} / \rho_{2}=0.9, k H_{1}=1.26, k H_{2}=6.28$, and $\varepsilon=0.03$. Red curve: linear theory. Blue curve: nonlinear theory from lower layer solution. Blue dots: nonlinear theory from upper layer solution.

Fig. 4. Particle trajectories at the interface for the same parameters as in Fig. 3. Red solid curve: upper layer. Blue solid curve: lower layer. The dotted red and blue curves correspond to the linear solution in each layer.

Fig. 5. The interface at the vertical wall $(b=0)$ when $\rho_{1} / \rho_{2}=0.9, k H_{1}=44, k H_{2}=0.63$, and $\varepsilon=0.022$. Red curve: linear theory. Blue curve: nonlinear theory from lower layer solution. Blue dots: nonlinear theory from upper layer solution. 